\title{
Results from the EROS Microlensing Survey
}

\author{
J.P. Beaulieu \\ Kapteyn Institute, Groningen, The Netherlands \\ H.J.G.L.M. Lamers, W.J. de Wit \\ SRON and Astronomical Institute, Utrecht, The Netherlands
}

\begin{abstract}
.
We present a status report of the original EROS and the on-going EROS-2 microlensing surveys, which were created to search for dark matter in the Galactic halo via microlensing effects on LMC/SMC stars. Microlensing surveys provide long-term systematic observations of millions of stars in both Clouds which yield a unique database of stellar photometry and variability. We review the results obtained on pulsating stars and detail the similarities and differences of the Magellanic Cloud Herbig $\mathrm{Ae} / \mathrm{Be}$ stars with their Galactic counterparts.
\end{abstract}

\section{Observations Made By the EROS Microlensing Survey}

EROS (Expérience de Recherche d'Objets Sombres) is a French collaboration between astronomers and particle physicists to search for baryonic dark matter in the Galactic halo by the microlensing (Paczyǹski 1986) of stars in the Magellanic Clouds (MCs). A compact object in the Galactic halo passing close enough to the line of sight to a background star in the Magellanic Clouds creates an increase in the apparent brightness of the star. This phenomenon occurs during a chance alignment of the observer, the deflector, and the background star. If one assumes a standard co-rotating halo, the time scale $\tau_{0}$ of a microlensing event is given by the relation $\tau_{0}=70 \sqrt{M / M_{\odot}}$ days where $M$ is the mass of the deflector. To be sensitive to a wide range of mass for compact objects $\left(10^{-7}-10^{-1} M_{\odot}\right)$ two complementary approaches have been developed.

The first approach involved photographic monitoring of 6.4 million stars over a $5 \times 5$ degree field using the ESO Schmidt telescope from 1990 to 1994 . When possible, the plates were taken once a night in $B_{J}$ and $R_{C}$. Isolated stars can be found down to magnitude 20 with typical photometric uncertainties of 0.3 mag.

The second approach was sensitive to low mass compact objects. Between 1991 and 1994 about 15000 CCD images were taken with two broad bandpass filters $B_{E}$ and $R_{E}$ of an area of $1 \times 0.4$ degrees centered on the bar of the LMC. An SMC field of 1 x 0.4 degrees was observed between 1993-1995 and about 6000 images were taken. Light curves of 270,000 stars exist with up to 48 points per night! 
EROS has been upgraded recently and entered the EROS-2 phase. EROS2 uses the dedicated Marly $1 \mathrm{~m}$ telescope at ESO La Silla. The prime focus is equipped with a focal reducer and a dichroic beam splitter with a mosaic of eight CCD $2048 \times 2048$ in each channel. The total field is $0.7 \times 1.4 \mathrm{deg}$. The data collection started in July 1996 for a baseline observing period of 4 years. EROS-2 observes about 20 million stars a night, and monitors the LMC, SMC, the Galactic Bulge and some fields in the spiral arms (with approximately a daily sampling rate).

\section{Microlensing Results}

The initial success of microlensing surveys was to show that the experiment was feasible. Indeed, the first microlensing events towards the LMC were announced almost simultaneously in October 1993 by the two competing teams EROS (Aubourg et al. 1993) and MACHO (Alcock et al. 1993, reported by Cook in this symposium). Now, about 18 events have been observed toward the LMC and the SMC. Despite the fact that some of the microlensing candidates turned out to be variable stars (EROS1 is a Be star, Beaulieu et al. 1995; EROS2 is an A0-2 eclipsing binary, Ansari et al. 1995; EROS-97-SMC-1, also known as MACHO-97-SMC-1, is an eclipsing binary, Palanque et al. 1998), the vast majority are most likely due to genuine microlensing. Moreover, we still do not know an intrinsic physical mechanism to mimic the observed photometric variation, and the microlensing interpretation is still favored.

EROS and MACHO measured consistent optical depths for gravitational lensing toward the LMC of $\tau_{L M C}=0.82_{-0.5}^{+1.1} 10^{-7}$ (Renault et al. 1997a) and $\tau_{L M C}=2.9_{-0.9}^{+1.4} 10^{-7}$ (Alcock et al. 1997), respectively. A subsequent combined EROS MACHO study gave $\tau_{L M C}=2.1_{-0.8}^{+1.3} 10^{-7}$ (Alcock et al. 1998). The expected optical depth to gravitational lensing towards the LMC due to known stellar populations is expected to be smaller than $0.510^{-7}$. The generally favored hypothesis is that all the observed microlensing are due to lenses in the halo. We should underline that in most cases, we do not have direct access to the different parameters of the system. The parameters are degenerate and therefore we do not know where the lens is (in halo or in the Clouds). Based on likelihood analysis it is concluded that half of the dark halo of our Galaxy is composed of MACHOs with a mass of $\sim 0.5 M_{\odot}$. If they are old white dwarfs it would lead to various serious problems involving the cooling rate of white dwarves, shape of the IMF for the first generation of stars, star formation rate, etc. If not, what are they? Do they belong to the halo?

On theoretical ground, Zhao (1998) had suggested the elegant idea of a tidal streamer front of the LMC as the cause of the microlensing signal. Zaritsky \& Lin (1997) claimed that a vertical extension of the red clump observed in LMC CMDs (VRC hereafter) was indeed the signature of such a streamer located at $35 \mathrm{kpc}$ from the Galaxy. Beaulieu \& Sackett (1998) confirmed the detection of the VRC, but showed that it could result from stellar evolution within the LMC, i.e. younger clump stars. The observed colors and densities in the CMDs are reproduced with a very simple model. Moreover, a radial velocity survey of VRC stars (Ibata, Lewis, \& Beaulieu 1998) showed that they have the same systemic velocity as the LMC field stars, and the same velocity dispersion. The chance 
of a tidal streamer having these kinematic properties are indeed low. The VRC is not the signature of a tidal streamer. However, it would be interesting to do an extensive search of tidal streamers very close to the LMC.

Recent studies towards the SMC by EROS (Palanque et al. 1998; Afonso et al. 1998) or by PLANET (Albrow et al. 1998) suggest that the events for which the degeneracy between the parameters have been partially broken are due to lensing in the Clouds themselves, not to halo lenses. The LMC and SMC self-lensing may have been systematically underestimated.

\section{Variable Star Results}

The microlensing surveys generate a very large homogeneous database of stars observed over a long period of time in the two Magellanic Clouds. They provide gold mines for stellar studies (see also results from the MACHO collaboration Welch et al., Alves et al. this volume). We will briefly review the results on pulsating variables, novae, and eclipsing binaries. Finally, we will present a status report of our study on pre-main sequence stars in the Magellanic Clouds.

\subsection{Pulsating Variables, Novae, Eclipsing Binaries}

We have systematically searched the EROS database for variable stars using the AoV method (Schwarzenberg-Czerny 1989) and have built catalogues of variable stars in the LMC and in the SMC. A differential study of about 550 Cepheids from LMC and SMC has been conducted to derive constraints on stellar pulsation theory (position of resonance centers and beat Cepheids) and to explore the subtle metallicity effect on the PL relation (Beaulieu et al. 1995, 1997a, 1997b; Sasselov et al. 1997). A larger sample of Cepheids from LMC and SMC has been observed as a pilot project of the EROS-2 survey (Bauer et al. 1998; Marquette, Graff, Ripepi et al. this volume).

The first results on $\sim 2000$ LMC RR Lyraes from EROS Schmidt plates has been presented by Hill \& Beaulieu (1997). The period histograms of RR Lyrae from the bar and from the field are similar. From the field they confirm the histograms found in the literature. The metallicity derived for these stars suggests that the old population in the field might have formed a little earlier than the oldest cluster in the LMC.

Grison et al. (1995) give a catalogue of 79 eclipsing binaries in the bar of the LMC ranging from close to well-detached type. These binaries can be used for accurate distance determination of the LMC or as a test of stellar pulsation models.

De Laverny et al. (1998) report the observation of a slow nova in the SMC. Due to the sampling rate of the observations ( 2 points/ $20 \mathrm{~min}$ ), low amplitude short time scale variation has been observed at maximum. This nova is a DQ Her type, similar to Galactic novae of the same class. A large helium enhancement in the shell is found, and $\mathrm{C}$ and $\mathrm{O}$ enrichments are suspected.

Beaulieu et al. (1997) and Kollàth et al. (1997) discuss the properties and compute linear and hydrodynamic models of a particular variable star observed in the bar of the LMC. It has 2 modes of pulsation coupled by a $3: 2$ resonance, shows a strong $H \alpha$ emission, and has a spectral type of late $\mathrm{O}$ to early $\mathrm{B}$. Its nature remains a puzzle. Is it a pre-main sequence star or post-AGB? 


\subsection{Pre-main Sequence Stars in the Magellanic Clouds}

The process of star formation of low mass stars and their subsequent evolution to the main sequence in external galaxies is essentially unknown. All the studies of star formation in external galaxies so far have been restricted to massive star clusters such as the 30 Dor region in the LMC. This is because massive young stars are very luminous and the HII regions are signposts of recent massive star formation. The population of low mass stars can be studied in such cluster by deep photometry. In this way, the low mass population of the 30 Dor and around SN1987A has been studied (see Panagia, Walborn, Zinnecker and references in this volume). However, such studies are always biased to conditions in massive star clusters which are not characteristic for the formation and the IMF of the majority of the less massive stars.

Using data from the EROS survey in the bar of the LMC, we discovered a population of Herbig $\mathrm{Ae} / \mathrm{Be}$ stars (HAeBe stars hereafter) on the basis of their characteristic irregular photometric variations and their colour. Subsequent optical spectra confirmed the spectral types $\mathrm{B}$, and the presence of strong $H \alpha$ emission. The first seven have been published by Beaulieu et al. (1996), Lamers et al. (1998), and the new 15 HAeBe stars by de Wit et al. (1999). The LMC $\mathrm{HAeBe}$ stars are located in a large star-forming region in the Bar with strong IR excess at IRAS wavelengths. Two stars with very similar characteristics were found in the SMC in a pilot search using EROS-2 data. These stars are the analogues of the Galactic pre-main sequence HAeBe stars.

The fields near the HAeBe stars are ideally suited for the study of the low mass star formation, because they contain many $\mathrm{T}$ Tauri stars. In fact, a study of these fields at ESO in BVRI and $\mathrm{H} \alpha$ down to $19.5^{\mathrm{m}}$ reveals colour-magnitude diagrams that contain hundreds of emission line objects (HAeBe and T Tauri stars) down to the detection limit. Only a few dozen emission lines objects are observed in the control fields outside the star forming region. The magnitudes of the optical study limit the determination of the IMF to $M_{v} \simeq+0.5^{m}$ or $M_{*} \simeq 3 M_{\odot}$.

The location of the Galactic PMS in the HRD shows an upper envelope called "the birth-line". It marks the location of the stars at the end of the fast accretion rate when they become optically visible through the dust. The birthline has been predicted for different accretion rates by Palla \& Stahler (1993) and Bernasconi \& Maeder (1996). There are no Galactic HAeBe stars with $M_{*}>10 M_{\odot}$, with the exception of a few stars which have uncertain distances. Five of the six HAeBe stars in the LMC studied in detail by Lamers et al. (1998) have luminosities above the observed and predicted Galactic birth-line by factors 3 to 10 (see Figure 1)! Does the birth-line depend on metallicity? If so, we should find a systematic trend from SMC, LMC to Galaxy. What could be the reason? Is the accretion rate dependent on metallicity? Faster accretion would result in a higher birth-line. Or does dust play a more crucial role than predicted in determining the luminosity of the star when its optical radiation emerges? (A lower metallicity may result in a smaller dust/gas ratio).

Galactic $\mathrm{HAeBe}$ stars show irregular photometric variations with amplitudes of typically a few tenths of a magnitude and occasionally a deep minimum up to 2 mag. Normally, Galactic HAeBe stars are "redder-when-fainter". This is explained by irregularity of the surrounding dust clouds orbiting the star, 

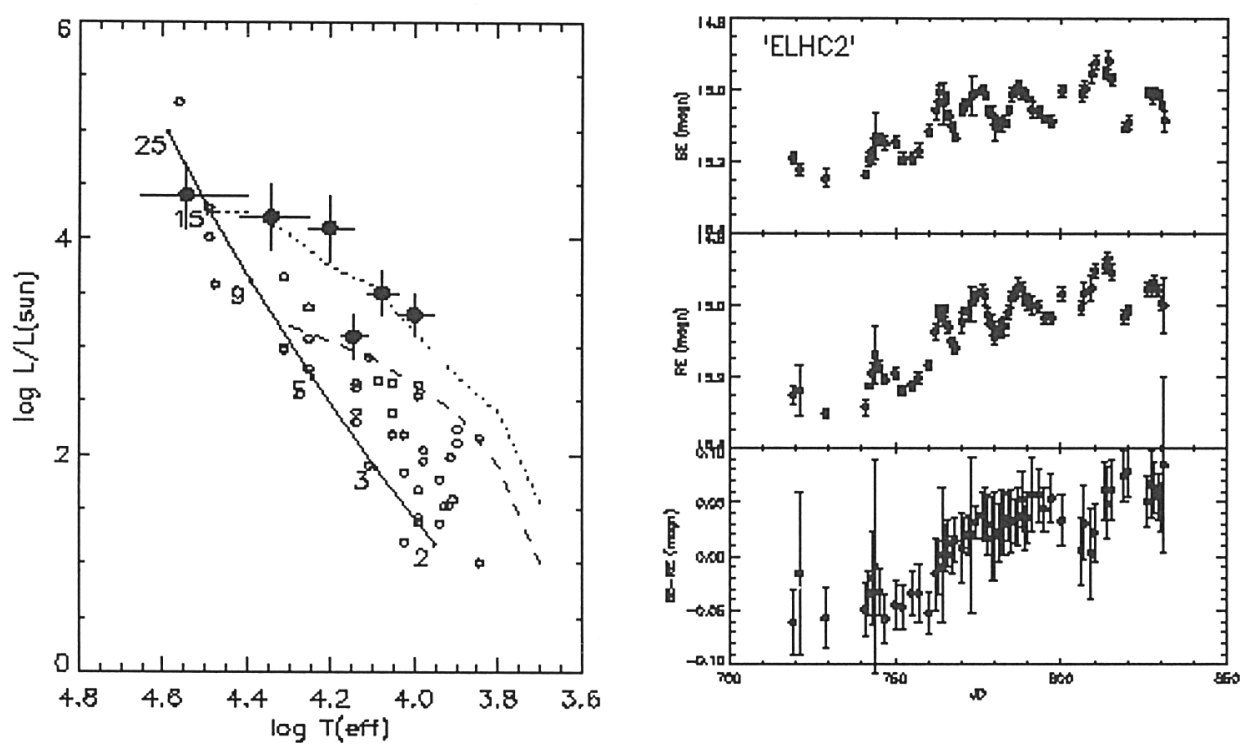

Figure 1. Left figure: The location of the first six LMC HAeBe stars in the HRD (points) with the Galactic HAeBe stars (circles). The LMC HAeBe stars are well above the galactic upper limit ("birth-line"). Dashed line: predicted birth-line for an accretion rate $10^{-5} M_{\odot} y r^{-1}$ and dotted line for $10^{-4} M_{\odot} y r^{-1}$ from Palla \& Stahler (1993). Right figure: The light curve and the colour curve of the LMC HAeBe star ELHC2. The time is in days since Jan 1 1990. Notice the irregular variations and the curious fact the star is "bluer-when-fainter". (From Lamers et al. 1998).

and confirmed by simultaneous photometric and polarimetric observations (e.g., Grinin 1994). The LMC and SMC HAeBe stars show a peculiar irregular colour magnitude variation: they are "bluer-when-fainter" (see star ELHC2 in figure 1). This reverse colour effect might be due to the contribution of a reflection nebula. The dust clouds that produce the light curves are small compared to the reflection nebula and may diminish the light of the star, while most of the (blue) scattered light from the reflection nebula is unattenuated. The net result is a decrease in brightness at all wavelengths, but slightly less in B than in V. This effect rarely occurs in observations of Galactic HAeBe stars, (only during very deep minima) because the photometric aperture is much smaller than the projected reflection nebulae.

We suggest that the bluing effects of LMC/SMC HAeBes is because we are observing the stars at $50 \mathrm{kpc}$, and therefore not resolving their associated reflecting nebulae. For instance, the reflecting nebulae around V380 Ori about 1 arcmin in diameter would be only 0.6 arcsec projected in the LMC. So the scattered blue light from the nebulae will contribute significantly to the EROS photometry. We have modeled this effect by scaling the photometric variations 
of the HAeBe star V380 Ori and its nebulae to a distance of the LMC. The "redder-when-fainter" behavior of V380 becomes a bluer when fainter behavior.

Acknowledgments. This work is based on observation held at ESO la Silla. JPB is grateful to IAU for financial support in attending IAU190, and to members of the EROS and PLANET collaborations.

\section{References}

Afonso, C., et al. (EROS coll.) 1998, A\&A, 337, L17

Albrow, M., et al. (PLANET coll.) 1998, ApJ, 509, 687

Alcock, C., et al. (MACHO coll.) 1993, Nature, 365, 621

Alcock, C., et al. (MACHO coll.) 1997, ApJ, 486, 697

Alcock, C., et al. (MACHO coll.) 1998, ApJ, 499, 9

Ansari, R., et al. (EROS coll.) 1995, A\&A, 299, L21

Aubourg, E., et al. (EROS coll.) 1993, Nature, 365, 623

Beaulieu, J.P., et al. (EROS coll.) 1995, A\&A, 299, 168

Beaulieu, J.P., et al. (EROS coll.) 1995, A\&A, 303, 137

Beaulieu, J.P., \& Sasselov, D.D. 1997, in Variable stars and the astrophysical return of microlensing surveys, eds Ferlet R. \& Maillard J.P., editions frontieres.

Beaulieu, J.P., et al. (EROS coll.) 1996, Science, 272, 995

Beaulieu, J.P., et al. (EROS coll.) 1997a, A\&A, 318, L47

Beaulieu, J.P., et al. (EROS coll.) 1997b, A\&A, 321, L5

Beaulieu, J.P., \& Sackett, P.D. 1998, AJ, 116, 209

Bernasconi, P., \& Maeder, A. 1996, A\&A, 307, 829

de Laverny, P., et al. (EROS coll.) 1998, A\&A, 335, L93

Grinin, V.P. 1994, in Nature and evolutionary status of Herbig Ae/Be stars ASP Conf Ser. Vol. 62, ed. P.S. Thé.

Grison, P., et al. (EROS coll.) 1995, A\&A, 109, 445

Hill, V. \& Beaulieu, J.P. 1997, in Variable stars and the astrophysical return of microlensing surveys, eds Ferlet R. \& Maillard J.P., editions frontieres.

Ibata, R.A., Lewis, G.F., \& Beaulieu, J.P. 1998, ApJ, 509, L29

Lamers, H.J.G.L.M., Beaulieu, J.P., \& de Wit, W.J. 1998, A\&A, 341, 827L

Palanque-Delabrouille, N., et al. (EROS-2 coll.) 1998, A\&A, 332, 1

Palla, F. \& Stahler, S.W. 1993, ApJ, 418, 414

Renault, C., et al. (EROS coll.) 1998a, A\&A, 324, L69

Renault, C., et al. (EROS coll.) 1998b, A\&A, 329, 522

Sasselov, et al. (EROS coll.) 1997, A\&A, 324, 471

Welch, D.L., et al. 1999 (MACHO coll.), this volume

Zhao, H.S. 1998, MNRAS, 294, 139

Zaritsky, D., \& Lin, D. 1997, AJ, 114, 254 


\section{Discussion}

Despina Hatzidimitriou: What is the status of analysing the EROS data for RR-Lyrae's in the SMC?

Beaulieu: We have light curves for 5.3 million stars in the SMC. We haven't done the absolute calibration yet. We haven't searched for RR Lyraes. In the LMC, we have catalogued 10,500 RR Lyraes among the 13 million stars.

Hans Zinnicker: Congratulations on your discovery of pre-main sequence stars in the Magellanic Clouds! Let me also refer you to the poster by Brandner et al. who use the technique of comparing deep CCD H $\alpha$ images with deep CCD R images to detect $\mathrm{H} \alpha$ emitting pre-Main sequence stars in the Magellanic Clouds, e.g. in NGC 346. 